\title{
Distributed coding of sound locations in the auditory cortex
}

\author{
G. Christopher Stecker, John C. Middlebrooks \\ Kresge Hearing Research Institute, University of Michigan, Ann Arbor, MI 48109-0506, USA \\ Received: 7 July 2003 / Accepted: 7 September 2003 / Published online: 12 November 2003
}

\begin{abstract}
Although the auditory cortex plays an important role in sound localization, that role is not well understood. In this paper, we examine the nature of spatial representation within the auditory cortex, focusing on three questions. First, are sound-source locations encoded by individual sharply tuned neurons or by activity distributed across larger neuronal populations? Second, do temporal features of neural responses carry information about sound-source location? Third, are any fields of the auditory cortex specialized for spatial processing? We present a brief review of recent work relevant to these questions along with the results of our investigations of spatial sensitivity in cat auditory cortex. Together, they strongly suggest that space is represented in a distributed manner, that response timing (notably first-spike latency) is a critical information-bearing feature of cortical responses, and that neurons in various cortical fields differ in both their degree of spatial sensitivity and their manner of spatial coding. The posterior auditory field (PAF), in particular, is well suited for the distributed coding of space and encodes soundsource locations partly by modulations of response latency. Studies of neurons recorded simultaneously from PAF and/or A1 reveal that spatial information can be decoded from the relative spike times of pairs of neurons particularly when responses are compared between the two fields - thus partially compensating for the absence of an absolute reference to stimulus onset.
\end{abstract}

\section{Introduction}

Intact auditory cortex is essential for normal soundlocalization behavior. That assertion is supported strongly by the localization deficits that arise following cortical lesions in humans (Greene 1929; Wortis and Pfeiffer 1948; Sanchez-Longo and Forster 1958; Klingon

Correspondence to: J. C. Middlebrooks

(e-mail: jmidd@umich.edu) and Bontecou 1966; Zatorre and Penhune 2001) and experimental animals (Heffner and Masterton 1975; Jenkins and Masterton 1982; Jenkins and Merzenich 1984; Kavanagh and Kelly 1987; Heffner and Heffner 1990). Little else about the role of auditory cortex in spatial hearing can be stated with such confidence. Many fundamental questions remain topics of ongoing investigation and debate: Are sound-source locations represented by restricted, local populations of neurons, or is the representation broadly distributed? Are specific cortical areas specialized for spatial processing, or do all areas make equivalent contributions? Is intracortical processing needed for accurate identification of soundsource locations, or is that task completed at subcortical levels? What features of neural spike patterns carry location-related information?

Unlike the cortical representation of visual space, which is passively inherited from the spatial topography of the retina, auditory space must be actively constructed from various monaural and binaural cues. This construction begins in the brainstem and, indeed, may be more or less complete at that level. Support for this view includes the presence of a systematic map of auditory space in the superior colliculus, where shortlatency responses appear to preclude cortical processing (Palmer and King 1982; Middlebrooks and Knudsen 1984). The results of lesion studies, however, suggest that the cortex plays a functional role in spatial processing. It is possible that the auditory cortex acts to integrate or further refine the representation of acoustic space initially generated by brainstem mechanisms. Alternatively, its role may be simply to distribute preprocessed spatial information to other systems. In the absence of strong evidence otherwise, we have argued for this more conservative interpretation.

Regardless of which spatial computations are actually performed within the auditory cortex, the responses of cortical neurons are clearly sensitive to manipulations of spatial location. In that sense, they can be said to represent auditory space - along with a number of other relevant stimulus dimensions such as intensity, frequency content, and temporal modulation. The manner 


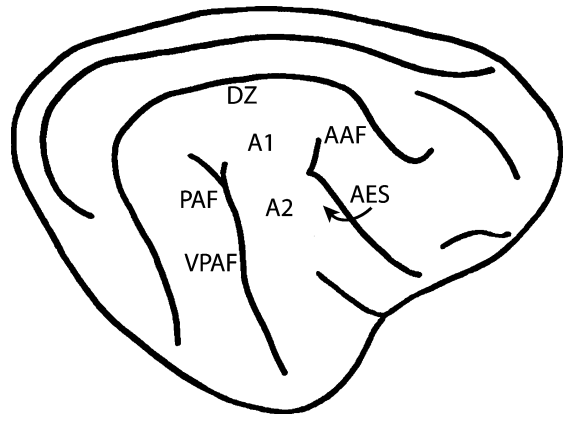

Fig. 1. Auditory cortical fields in the cat. A view of the sulcal pattern of a cat's right cortical hemisphere, showing the relative locations of the primary (A1), second (A2), anterior (AAF), posterior (PAF), and ventral-posterior (VPAF) auditory fields, along with the dorsal zone (DZ) and the field of the anterior ectosylvian sulcus (AES)

in which such features are represented and the ways in which multiple representations are combined in the responses of cortical neurons are of interest to both computational neuroscientists and sensory scientists. Work in our laboratory has focused on questions of how auditory space is represented by cortical neurons, how such representations might be decoded by "downstream" neurons, and how spatial coding strategies might vary between different cortical fields. Answering these questions is of fundamental importance to understanding the way in which cortical structures form representations of the outside world. In this paper, we will present evidence from the cat's auditory cortex that sound-source locations are represented in a distributed fashion, we will describe two cortical areas that seem to show specialization for spatial processing, and we will demonstrate that relative spike timing among cortical fields can carry important location-related information.

The auditory cortex of cats and primates is composed of a number of anatomically and physiologically distinct subfields that can be divided into a primary "core" (field A1) and a surrounding "belt" of higher-order fields, including (in the cat, see Fig. 1) the second auditory field (A2), the field of the anterior ectosylvian sulcus (AES), anterior, posterior, and ventral-posterior auditory fields (AAF, PAF, and VPAF), and a dorsal zone (DZ) (Rose and Woolsey 1949; Reale and Imig 1980; Middlebrooks and Zook 1983; Rouiller et al. 1991; Huang and Winer 2000).

Compared to the core, fields in the belt have higher proportions of neurons with broad and/or complex frequency tuning, long and stimulus-sensitive response latencies, and diverse spatial tuning. Our studies of spatial sensitivity have focused on fields A1 (Stecker et al. 2003b), A2 (Furukawa and Middlebrooks 2001), AES (Middlebrooks et al. 1998; Xu et al. 1998), PAF (Stecker et al. 2003b), and DZ (Stecker et al. 2003a). In the majority of these studies, we have observed similar spatial sensitivity throughout the auditory cortex and have argued against the existence of fields specialized for spatial processing. Our recent studies of spatial sensitivity in PAF and DZ, however, reveal quantitative improvements in spatial sensitivity over that seen in other fields.
More importantly, they reveal key differences in the way various belt fields represent auditory space - differences that might have important functional consequences.

\section{Local vs. distributed codes}

In general, there are two types of strategies for representing information in neural populations. These form the ends of a continuum based on the stimulus specificity of individual neurons. On the one hand is the local strategy in which each of a large number of highly specific (or "sharply tuned") neurons responds optimally to a very narrow range of stimuli. This strategy provides many advantages - primarily ease of decoding - and conforms to Müller's doctrine of specific nerve energies. One need only know which neuron (or channel) is active to know which stimulus is present. A clear example of a local code is the representation of visual space among sensory receptors in the visual system, where individual receptors respond to stimulation of small patches of the retinal surface. This local representation is propagated throughout the visual system such that cortical neurons maintain selectivity for the retinal position of a stimulus. Moreover, subsequent stages of visual processing maintain a retinotopic arrangement of receptive fields, so that neighboring cortical neurons respond to input from neighboring regions of visual space. Similar topographic or map-like representations are also seen in the somatotopic projection from the skin surface to somatosensory cortex and the cochleotopic projection (representing stimulus frequency) from the inner ear to auditory cortex. Indeed, topographic organization appears to be a general feature of local representations in the central nervous system.

The distributed strategy, on the other hand, encodes information by the joint response of many neurons. The responses of individual neurons are not specific to particular stimuli but are graded, being strongest for preferred stimuli and weaker (but still present) for lesspreferred stimuli. Distributed representations are generally more compact, requiring fewer neurons than local representations, but require more complex decoding because the responses of individual neurons are ambiguous outside the context of the population. Distributed codes are also more robust to trauma and, because they use relative rather than absolute activity levels, can maintain fairly constant output across a range of stimulus intensities or background-activity levels (Hinton et al. 1986). An example of this type of strategy is the representation of color information by photoreceptors in the retina (DeValois and DeValois 1993). Cones differentially sensitive to short, medium, or long wavelengths each respond to a wide range of stimulus wavelengths, so that the response of a single cone type confounds color information with stimulus intensity. Comparing the responses of multiple cone types, however, provides for acute discrimination of wavelength and avoids some of the difficulties in distinguishing changes in color from changes in overall stimulus intensity. As a result, three graded channels of infor- 
mation are sufficient to encode a vast array of discriminable colors. Other examples of distributed representations can be found in the motor cortex - where reaching responses are encoded by large populations of broadly tuned neurons that "vote" for their preferred movement directions (Georgopoulos et al. 1986) - and in the olfactory and chemosensory systems, where information is carried by patterns of activation across a limited set of receptor types. In general, it seems that distributed representations are employed by the cortex wherever the number of receptor or effector channels (the underlying input or output dimensionality) is limited.

Input to the auditory system originates in the cochlea, where acoustic frequency is mapped in a continuous manner along the sensory epithelium. As mentioned in the introduction, however, spatial processing involves the extraction of spatial cues by brainstem mechanisms; input to cortical structures involved in the transmission or further processing of spatial information derives from these brainstem nuclei. It now appears that brainstem representations of spatial cues involve a limited number of spatial "channels" rather than continuous maps. In an elegant and long-standing model of binaural processing, Jeffress (1948) proposed that interaural time differences (ITD, a primary cue for sound-source azimuth) are computed by arrays of neurons tuned for particular values of interaural delay (i.e., using a local representation of ITD). In disagreement with that view, David McAlpine and colleagues have recently demonstrated that the inferior colliculus (IC) and medial superior olive (MSO, the brainstem nucleus most strongly implicated in ITD processing) contain a preponderance of neurons favoring particular values of ITD directly related to their preferred frequencies (Brand et al. 2002; McAlpine et al. 2001). Moreover, the preferred ITDs of many low-frequency neurons lie outside the range of behavioral relevance, an impractical feature for local representation of ITD. The slopes of ITD-tuning functions, however, are generally steepest (i.e., most sensitive) across the interaural midline, where behavioral acuity is highest, and McAlpine et al. (2001) argue that best ITDs are arranged to position the ITDtuning slopes appropriately. Thus, the representation of ITD in the brainstem appears to involve a limited number of ITD-sensitive channels (Marquardt and McAlpine 2001), adopting a distributed - not local representational strategy. By analogy to other systems, we might therefore expect to find a distributed representation of auditory space in the cortex as well.

What physiological features of auditory cortical responses would we expect to see if cortical neurons adopted a local or distributed coding strategy for auditory space? Local coding requires neurons with narrow spatial tuning functions that sample space evenly (but with increased density in regions of high acuity such as the frontal midline). Based on the prevalence of topographic organization among local coding systems, we might also expect to find a spatiotopic arrangement of receptive fields (a "map" of auditory space) in the auditory cortex comparable to the cortical map of visual

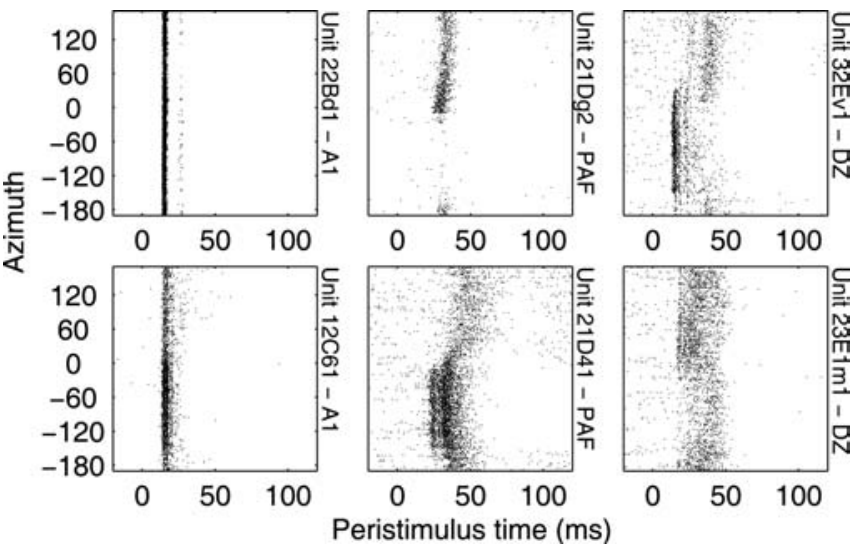

Fig. 2. Examples of individual unit responses typically seen in A1 (left), PAF (middle), and DZ (right). Each frame plots the peristimulus times ( $x$-axis) of spikes elicited by stimuli varying in location ( $y$-axis). In all three fields, response magnitudes (spike counts) are strongly modulated by stimulus location. In PAF and DZ - but not in A1 response latencies are also modulated by location. This modulation of response timing provides an additional means for units to encode spatial locations of sound sources and is responsible for increased spatial information transmission (at least in PAF; Stecker et al. 2003b) over that seen in A1

space. Distributed coding, on the other hand, requires neurons with broad, overlapping tuning functions covering auditory space. In the distributed case, the centers of neural tuning functions need not sample space evenly; a few broad "spatial channels" may suffice for accurate coding throughout $360^{\circ}$ of azimuth. Regions of high acuity would correspond to locations where responses undergo rapid changes (i.e., where the slopes of tuning functions are steepest and the relative activity of differently tuned neurons is most informative). Since auditory localization is most accurate near the interaural midline, a distributed strategy would likely position receptive fields such that tuning functions cross the midline with steep slopes (i.e., the receptive field peaks will be located away from the midline, not near it, as expected from a local strategy).

Although the notion of topographic maps for auditory space within the cortex seems reasonable based on analogies to spatial processing in the visual cortex and superior colliculus, numerous studies looking for local, map-like representations in the auditory cortex have failed to find them. Instead, the available data support the existence of distributed spatial representations in the auditory cortex. Figure 2 shows examples of spatial sensitivity of units in cortical areas A1, PAF, and DZ in the cat. The spatial tuning of individual cortical neurons is generally very broad - typically spanning $60-180^{\circ}$ of the contralateral hemifield - and systematic traverses across the cortex do not reveal systematic shifts in the spatial positions of receptive fields. Moreover, the distribution of preferred locations in the auditory cortex is not uniform. Rather, the majority of neurons tend to favor spatial locations lateralized toward the contralateral ear ( $\sim 40-90^{\circ}$ lateral to the interaural midline), with a substantial minority in some fields preferring ipsilateral locations (Fig. 3). This arrangement is similar to the 


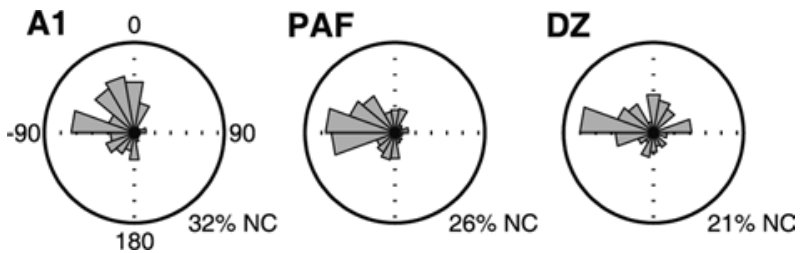

Fig. 3. Distributions of azimuth centroids (preferred locations in azimuth, based on stimuli $40 \mathrm{~dB}$ above unit thresholds) are plotted for A1 (left), PAF (middle), and DZ (right). "NC" values indicate proportions of nonselective units for which no centroids could be calculated. An overrepresentation of contralateral (negative) azimuths can be seen in all three distributions and is typical of auditory cortex. Especially in PAF and DZ, however, substantial minorities of units favor ipsilateral azimuths. In contrast to A1 and PAF, where more units prefer a range of intermediate locations around $40-60^{\circ}, \mathrm{DZ}$ includes larger proportions of units preferring lateral locations (around $\pm 90^{\circ}$, where interaural differences are presumably largest)

distribution of best ITD among neurons in the IC and MSO (McAlpine et al. 2001; Brand et al. 2002) and provides for maximum spatial resolution across the interaural midline, based on a distributed representation of azimuth.

Although continuous spatiotopic maps are not clearly present in any auditory cortical field so far studied, it is fairly common to observe groups of neighboring units (within $\leq 1 \mathrm{~mm}$ ) with similar spatial tuning. When recording in DZ using 16-channel electrodes oriented to enter the ventral bank of the suprasylvian sulcus, we often observed alternating regions $(450-1200 \mu \mathrm{m}$ in width) of ipsilateral or contralateral tuning (Fig. 4). Midline-tuned neurons were occasionally observed in between these regions. We hypothesize that these regions of lateral preference represent a continuation of the system of binaural bands observed in A1 (Middlebrooks et al. 1980). Binaural bands, oriented rostrocaudally and roughly orthogonally to the isofrequency contours of A1, consist of neurons with shared sensitivity to binaural interaction (e.g., excitation by contralateral stimulation and either excitation or inhibition by ipsilateral stimulation). Adjacent bands differ in their binaural sensitivities. If correct, our observations in DZ suggest that this banding structure continues into the most dorsal regions of the auditory cortex. The strong alternating structure is reminiscent of ocular-dominance columns in primary visual cortex; in this case, however, alternating ear dominance corresponds to alternating preference for regions of space, whereas ocular-dominance columns reflect alternating input channels carrying information about similar spatial locations. It is more reasonable to consider the alternating pattern as a coarse spatial "map" - albeit one based on a limited number of spatial "channels" (contralateral, ipsilateral, midline) rather than uniformly distributed and spatially arranged narrow receptive fields.

\section{Rate vs. temporal codes}

Studies of cortical processing have traditionally examined the effects of stimulus manipulations on response
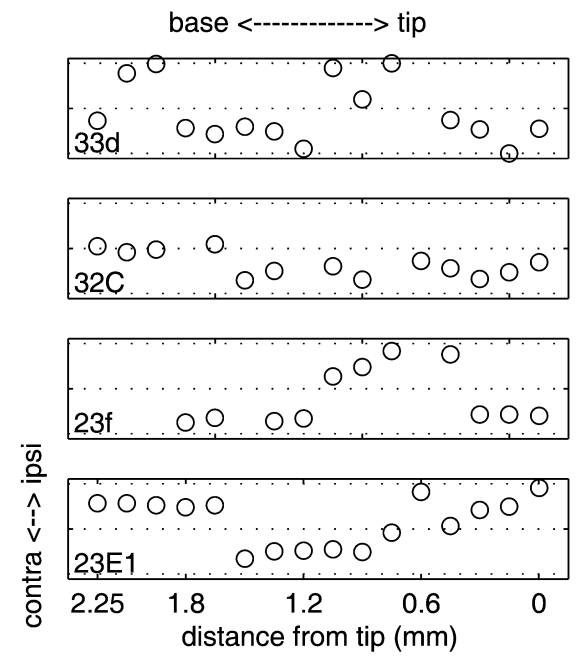

Fig. 4. Examples of the arrangement of preferred locations across the cortical surface in field DZ. Each panel depicts the azimuth centroids ( $y$-axis) of units recorded simultaneously on different channels of a single probe oriented lateromedially into the ventral bank of the suprasylvian sulcus. In all cases, sites near the probe tip recorded from medial locations (in an unfolded cortex, this would be the farthest dorsal from A1), while sites near the base recorded from more lateral locations (nearer to the A1/DZ boundary). Centroids are plotted as a function of distance along the probe. Consistent with our previous findings in other fields, many probe placements fail to reveal any systematic arrangement of spatial tuning. Several, however, revealed alternating ipsilateral and contralateral preference reminiscent of the arrangement of binaural bands in A1 (Middlebrooks et al. 1980)

magnitudes (spike counts or firing rates). Certainly, functions relating overall firing rates to relevant stimulus dimensions (e.g., sound level or tone frequency) provide the clearest means of determining neuronal tuning properties (receptive fields). A number of difficulties associated with decoding such "rate codes" (especially for auditory cortical neurons that respond with one or two spikes per stimulus), however, have prompted researchers to look for other information-bearing features of neural responses such as the timing of neural action potentials, their temporal dispersion, and dynamic changes in firing rate. Pattern-recognition analyses of such features reveal that, despite being broadly tuned for space, the responses of individual cortical neurons in A2 and AES can accurately encode spatial location with median errors as small as $25-40^{\circ}$ and information rates of up to 1.3 bits per stimulus (Middlebrooks et al. 1998; Furukawa and Middlebrooks 2001). As shown in Fig. 5, similar results are obtained in A1 and PAF, although PAF units transmit significantly more information about space (in azimuth and elevation) than units in A1 and appear to be the most accurate spatial coders among units in all fields studied so far (Stecker et al. 2003b).

Among information-bearing features of neural responses, first-spike latency is particularly robust and (theoretically) easy to decode, particularly when only one spike is elicited per stimulus. Spike timing in the auditory cortex is sensitive to sound-source location and carries as much or more spatial information than does 

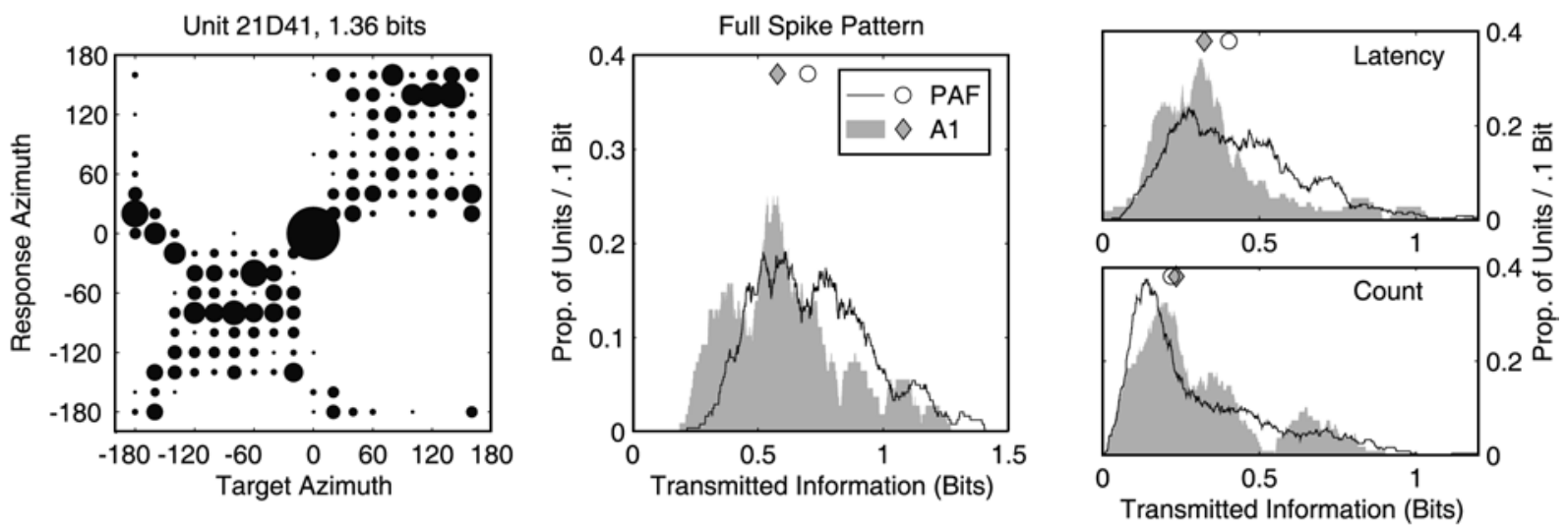

Fig. 5. Estimating spatial information carried by neural spike patterns. A statistical pattern recognition algorithm (see Stecker et al. 2003b for details) classifies each neural response according to the most likely eliciting stimulus location. Left panel: algorithm performance for one unit in PAF, represented by a joint stimulus-response matrix (confusion matrix). Proportions of responses at each combination of target ( $x$-axis) and response ( $y$-axis) location are indicated by the parameter of the circles inside the figure. In this case (rasters for this unit are shown in Fig. 2), classification is highly accurate between hemifields: contralateral targets (negative azimuths) are almost never misclassified to ipsilateral locations or vice versa. Targets on the midline are accurately localized, although discrimination of front from

spike rate (Brugge et al. 1996; Eggermont 1998; Furukawa and Middlebrooks 2001; Jenison 2001). While the two measures are often strongly correlated (effective stimuli tend to elicit short-latency, high-rate responses), they are not always totally so and may be used by some neurons in PAF to differentially encode various stimulus features (Stecker et al. 2003b).

PAF neurons respond with long and stimulus-sensitive latencies. This aspect of PAF responses forms the basis of a robust temporal code for auditory space and provides an added coding dimension, beyond spike rate, for neural representation. In contrast, A1 neurons respond with short latencies that vary only minimally between stimulus locations. Figure 6 plots distributions of overall latency and the range of spatial modulation of latency across units in A1, PAF, and DZ. Neurons in DZ, despite having shorter overall latencies than those in PAF, exhibit spatial modulation of response timing that is quite similar. Figure 5 (right panels) plots distributions of spatial information carried by the spike counts or response latencies of individual units in A1 and PAF. Whereas spike counts of units in the two fields transmit similar quantities of information, firstspike latencies transmit significantly greater spatial information in PAF than A1 (Stecker et al. 2003b). Moreover, PAF's improved spatial coding by response latency is responsible for that field's better overall spatial coding. Stecker et al. (2003b) found latencybased information rates to be highly predictive (accounting for $78 \%$ of between-unit variance) of full-pattern information rates in PAF, whereas spikecount-based information rates were less predictive (accounting for $58 \%$ of variance in full-pattern information; the overlap reflects redundancy in the two measures). In $\mathrm{A} 1$, latency and count were similar back is poor. Mutual information of target and response gives an estimate of the total stimulus-related information contained in the neural response; in this case, 1.36 bits. Center panel: distributions of spatial information for fields A1 (gray) and PAF (solid line). Symbols indicate median values in each field. Overall, units in PAF transmit significantly more information than units in A1. Right panels: distributions of spatial information carried by spike count (lower panel) or response latency (upper panel) alone. Units in the two fields transmit similar amounts of information by spike count, but PAF units transmit significantly more information by latency than do A1 units. Figure reproduced in part from Stecker et al. (2003b), with permission

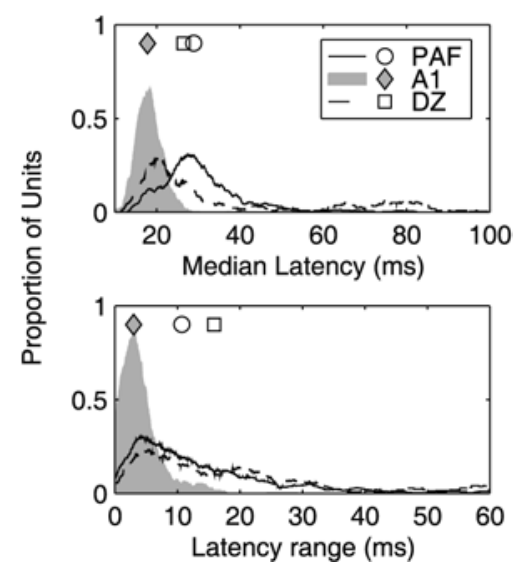

Fig. 6. Median latency and latency range in A1, PAF, and DZ. Top: Distributions of median latency (the median, across stimulus location, of geometric-mean first-spike latencies at each location) are plotted for fields A1 (gray), PAF (solid line), and DZ (broken line). Stimuli were presented $40 \mathrm{~dB}$ above unit thresholds. Symbols indicate median values in each field. Though skewed with long tails of late-responding units, the distributions follow an orderly pattern with A1 units responding most rapidly (overall median: $18 \mathrm{~ms}$ ), followed by DZ (27 ms, but note the multimodal distribution of latencies in that field) and PAF (29 ms). Bottom: Similar distributions are plotted for latency range (spatial variation of location-specific geometric-mean first-spike latencies). Consistent with the examples shown in Fig. 2, response latencies in fields PAF and DZ are strongly modulated by stimulus location. (Median latency range: A1 $3.0 \mathrm{~ms}$, PAF $10.7 \mathrm{~ms}$, DZ $15.9 \mathrm{~ms})$

predictors of full-pattern performance (accounting for $63 \%$ and $69 \%$ of variance, respectively).

Despite the significant quantities of spatial information transmitted by first-spike latency, however, spatial coding by latency is limited in one important regard - the 


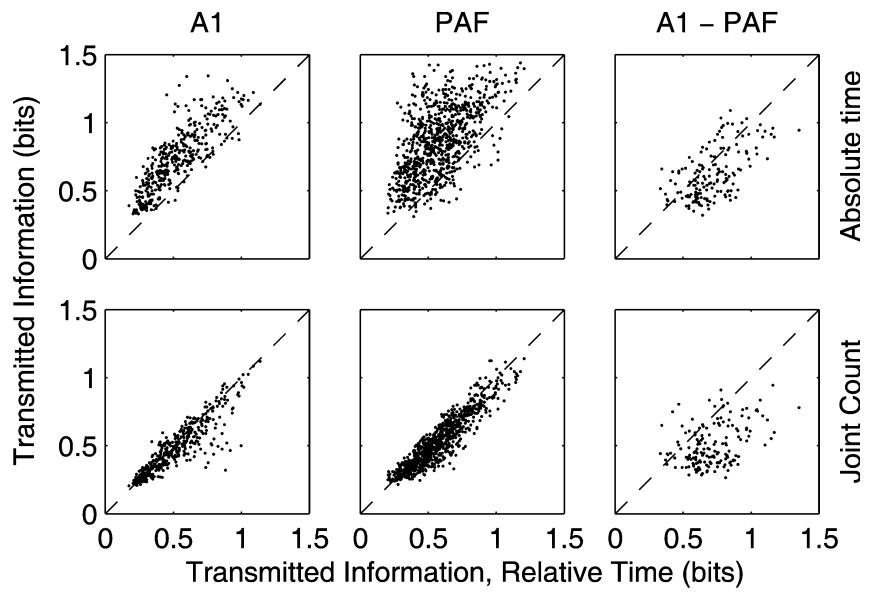

Fig. 7. Spatial information transmitted by relative spike times among pairs of simultaneously recorded units in A1 and/or PAF. The top panels plot information carried by full spike patterns, which preserve features of absolute spike timing ( $y$-axis) against information carried by relative spike times ( $x$-axis). The three panels are formatted identically and correspond to pairs of units recorded within field A1 (left), within field PAF (middle), or between fields (i.e., one unit in A1 and another simultaneously recorded in PAF, left). In both A1 and PAF, absolute spike timing transmits more information than relative times only. When spike times are compared between the two fields, however, relative timing is as informative as absolute timing. The lower panels plot, for the same fields, information carried by joint spike count ( $y$-axis) against information carried by relative time $(x$ axis). Again, between-field timing is especially informative. See Sect. 5 for measurement and algorithmic details

decoding of latency-based information requires an independent reference to stimulus timing, and cortical neurons do not have access to any such reference. Thus, there is no theoretically plausible means for the nervous system to compute the absolute latency of a cortical response. It is possible, however, that pairs (or groups) of neurons might encode spatial information in the relative timing of their responses such that a "downstream" neuron might recover sound-source locations by measuring the intervals between spikes of different neurons (Furukawa et al. 1998; Jenison 2001). As shown in Fig. 7, this relative-timing strategy is clearly less effective than using absolute latency, but it does convey usable information about auditory space, especially among PAF units. When the spike times of PAF units are compared to those of A1 units, however, the relative-time strategy is as effective as absolute time. In that case, A1 units' lack of space-dependent latency variation is advantageous because the timing of A1 responses provides a reference for the timing of PAF responses. The result suggests that a third brain structure receiving input from both fields could reconstruct space from time relatively effectively (Stecker et al. 2003a), without an absolute temporal reference.

As discussed above, spatial modulation of response latency is a potent spatial-coding feature of PAF neurons (and, it seems, for DZ neurons as well). Although we do not know the source of this modulation, it does not simply reflect differences in the effective level of stimulation (at the ear) associated with different sound-source locations (Stecker et al. 2003b). Rather, it reflects specific sensitivity of response timing to binaural or other spatial acoustic cues. Likewise, we do not fully understand the origin or functional significance of PAF's long overall latencies. They are not likely to reflect synaptic delay of input arriving via A1, as PAF receives its own parallel input from the auditory thalamus (ventral MGB), and responses in PAF are not dramatically affected by lesions to ipsilateral A1 (Kitzes and Hollrigel 1996). Instead, we have suggested that long latencies in PAF may reflect early inhibitory processes that act to delay responses, especially those elicited by nonoptimal stimuli. In addition to creating a robust neural code for space, these delays may play a role in multisensory integration by temporally synchronizing auditory cortical outputs with the responses of cortices representing other sensory modalities that operate at a slower time scale (Stecker et al. 2003b).

\section{Cortical specialization for spatial processing}

It has recently been suggested - by analogy to the monkey visual cortex - that the auditory belt cortex of the monkey might be composed of independent functional and anatomical "streams" specialized for the processing of spatial and spectral information (Rauschecker 1998; Rauschecker and Tian 2000). Much of our recent research has focused on identifying differences in spatial sensitivity between various auditory cortical fields in the cat, with the goal of identifying, if possible, fields that are specialized for spatial processing. The presence or absence of such fields bears strongly on the correctness of the "streams" view, at least for the cat model.

A number of features of responses in cortical field PAF of the cat suggest that PAF may indeed be specialized for the distributed representation of auditory space. As described in Sect. 3, PAF responses carry more space-related information than do A1 responses and do so by making enhanced use of a second coding dimension - response timing - beyond spike rate. Spatial tuning, although still very broad, is somewhat sharper in PAF than in A1. More importantly, PAF neurons tend to maintain their spatial tuning at high stimulus levels, whereas tuning widths of A1 neurons broaden considerably with increasing sound level ${ }^{1}$. This factor may help neurons in PAF to accurately signal sound locations with invariance to overall stimulus intensity. Relative to A1, PAF contains larger proportions of neurons favoring ipsilateral azimuths, and best elevations are distributed more evenly among PAF units as well. Thus, it can be said that PAF samples space more evenly than does A1, and this diversity - coupled with the availability of latency coding in PAF responses - plays a role in the distributed representation of auditory space by ensembles of

\footnotetext{
${ }^{1}$ It is interesting to note, however, that the deleterious effects of increasing stimulus levels on the spatial tuning of A1 neurons seem to be diminished in awake cats (Mickey and Middlebrooks 2003; Stecker et al. 2003b).
} 
PAF neurons. Information-theoretic analyses reveal that ensembles of PAF neurons transmit more spatial information than do ensembles of A1 neurons (as expected based on the increased spatial information of PAF units overall). Ensemble coding in PAF also benefits more from increases in ensemble size such that PAF ensembles of 16-32 units are much better at encoding space than are A1 ensembles of the same size. Recalling that the spatial receptive fields of $\mathrm{A} 1$ neurons are generally confined to contralateral space whereas the population of PAF neurons includes more ipsilaterally tuned units, it seems that the ability of PAF to accurately represent space in a distributed fashion may depend on its diversity of spatial tuning (Stecker et al. 2003b). This is a key feature of PAF responses that sets it apart as a strong contender for specialized spatial processing.

In every respect, then, PAF neurons appear better suited for spatial processing than neurons in A1 or other studied fields (e.g., A2, AES) - with the possible exception of DZ, which shares with PAF a number of features including level independence and spatial modulation of response timing (Stecker et al. 2003a, see also Fig. 6). Nevertheless, each of these differences is a matter of degree rather than a clear (qualitative) difference in spatial sensitivity. To be sure, spatial modulation of response latency in PAF represents a qualitative difference in the manner in which stimuli are encoded in that field, but no physiological feature identifies PAF neurons as uniquely - obviously - spatial. Without clear differences of that sort, claims regarding the functional role of PAF (or any other cortical field) in spatial hearing must be addressed through different means. Lesion studies of behaving animals, in particular, may be used to identify brain regions essential to spatial auditory tasks. Through reversible-lesion studies using cortical cooling, Malhotra et al. (2003) have recently identified PAF among auditory cortical fields necessary for sound-localization behavior in cats. In their study, unilateral deactivation of PAF, A1, or AES produced profound deficits in contralateral auditory (but not visual) localization, whereas cooling other areas (including A2, AAF, and VPAF) had no effect on sound localization. Together with our results, this suggests that PAF may indeed play an important role in spatial hearing. As mentioned in introduction the Sect. 1, however, that role might only be to distribute auditory spatial information to multisensory and motor areas.

\section{Methods}

The methods of animal preparation, experimental apparatus, and procedures used for this report have been described in detail previously (Stecker et al. 2003b). Briefly, we recorded extracellular neural activity from the right auditory cortex in ten anesthetized adult cats while presenting broadband acoustic stimuli from various free-field locations. All procedures complied with the guidelines of the University of Michigan Committee on Use and Care of Animals.
Unit recording and signal processing. We recorded unit activity in cortical fields A1 (257 units), PAF (679 units), and DZ (354 units), which were identified initially by the arrangement of cortical sulci and subsequently by the physiological responses observed in each recording. Field A1, located ventral to SSS and rostral to PES, was characterized by short-latency responses with sharp frequency tuning and caudorostral upward progression of best frequencies. Field PAF, lying mostly within the caudal bank of PES, was characterized by long-latency responses, multiple peaks of frequency tuning, and nonmonotonic responses to increasing sound level. Field DZ, extending dorsally from A1 into the ventral bank of SSS, was characterized by broader and/or multipeaked frequency tuning substantially different from that observed in neighboring regions of $\mathrm{A} 1$.

Extracellular activity was recorded using one or two multichannel microelectrode arrays ("Michigan probes") manufactured by the University of Michigan Center for Neural Communication Technology (Anderson et al. 1989). These fixed-geometry arrays allowed simultaneous recording from up to 16 sites per probe (up to 32 sites overall). Site spacing ranged from 100 to $200 \mu \mathrm{m}$ between electrode sites and varied between different probe types. Activity at each site was bandpass-filtered $(0.2-4 \mathrm{kHz})$ and stored on computer disk for offline analysis, including removal of correlated noise (Bierer and Anderson 1999), spike detection, and spike sorting by principal-components analysis. By our most conservative criteria for unit isolation (Furukawa and Middlebrooks 2001), roughly $5 \%$ of recordings could be reliably classified as isolated single neurons. The remainder were either clusters of two or more neighboring neurons or single units whose spikes varied in shape due to low signal-to-noise ratio. Consistent with previous experience, we observed no systematic differences between the tuning properties of isolated single neurons and those that were less well isolated. Throughout this report, we use the term "unit" to refer to both.

Stimulus presentation and data analysis. Cats were suspended in the center of a $2.6 \times 2.6 \times 2.5-\mathrm{m}$ soundattenuating chamber whose surfaces were lined with sound-absorbing foam to suppress acoustic reflections. Sounds were presented one at a time from calibrated loudspeakers $1.2 \mathrm{~m}$ from the cat's head and spaced $20^{\circ}$ apart in the horizontal plane. Loudspeaker locations are expressed in degrees azimuth, relative to directly in front of the cat $\left(0^{\circ}\right)$. Positive azimuths correspond to the cat's right side (ipsilateral to the recording site). Acoustic stimuli were 80-ms Gaussian noise bursts with abrupt onsets and offsets, presented randomly from locations in the loudspeaker array at levels $20-40 \mathrm{~dB}$ above unit threshold. Unit recordings for each stimulus were 100$250 \mathrm{~ms}$ in duration, including a prestimulus period of 20-50 ms used for estimation of correlated noise sources (Bierer and Anderson 1999) and determination of spikedetection thresholds. 
Units that failed to respond to their most preferred stimulus with at least one spike per trial on average were eliminated from further analysis, as were units whose mean spike counts varied by a factor of two or more between the first and second half of the series of trials. After screening, we analyzed the responses of 210 units in A1, 411 units in PAF, and 235 units in DZ. Sorted spike times were stored as latencies $(10-\mu$ s resolution) relative to the onset of sound at the loudspeaker. From these we computed azimuth and elevation centroids (a measure of preferred location), spatial tuning widths, and stimulus-dependent response latencies (geometricmean first-spike latency) for each unit, separately at each tested level (see Stecker et al. 2003b for details).

Analysis of spatial information carried by relative spike times. We assessed the transmission of spatial information by relative spike times of 1382 pairs of simultaneously recorded units (Fig. 7) using a pattern recognition algorithm similar to that used previously to study the responses of individual neurons and neural ensembles (Stecker et al. 2003b, see also Fig. 5). Briefly, three conditions were tested. In the first condition ("absolute time + joint count"), ensemble spike patterns were generated by computing bootstrapped spike-density functions (SDFs) for each unit. These are spike times recorded on eight randomly selected trials corresponding to a particular stimulus location (stimulus levels 20-40 dB above threshold were included), convolved with a Gaussian impulse $(\sigma=1 \mathrm{~ms})$, and resampled to produce a histogram of spike count per 2-ms bin. The bootstrapping procedure used identical trials for the two units, and the two resulting SDFs were concatenated to produce a single vector-form input pattern. The procedure was repeated to produce 20 different input vectors per stimulus location. In the second condition ("relative time + joint count"), we computed all first-order between-unit interspike intervals (ISIs) for each trial. In that case, ISIs were the (signed) intervals between successive spikes recorded from the two units under consideration. The computation of ISIs for each trial was followed by bootstrapping, convolution, and resampling - as described for the first condition - to generate input vectors. In the third ("joint count") condition, input vectors were generated as in the relative time condition, but the actual ISI values were randomly permuted across the entire set of ISIs. In that way, the number of intervals (which provides information about spike count) elicited by each stimulus was preserved, as was the global distribution of relative spike times, but stimulus-related modulation of relative spike times was eliminated. Input patterns generated in each condition, for each pair of units, were classified by a template-matching algorithm based on input patterns generated from an independent "training" set of trials. Each input pattern in the test set was classified according to the most-likely stimulus location to have elicited the neural response. Classifications were expressed in joint stimulus-response matrices (confusion matrices; see Fig. 5, left panel), from which we calculated the total stimulus-related information (mutual information), in bits (Furukawa and Middlebrooks 2001).

Acknowledgements. We thank Ian Harrington, Ewan Macpherson, and Brian Mickey for assistance with data collection and Zekiye Onsan for technical and administrative support. Ian Harrington generated the cortical diagram in Fig. 1; he and Ewan Macpherson also provided helpful comments on earlier versions of this manuscript. Funding for this research was provided by the National Institutes of Health (NIH R01-DC-00420, T32-DC-00011, P01DC-00078 P30 DC0S188) and the National Science Foundation (NSF DBI-0107567). Recording probes were provided by the University of Michigan Center for Neural Communication Technology (NIH P41-RR-09754).

\section{References}

Anderson DJ, Najafi K, Tanghe SJ, Evans DA, Levy KL, Hetke JF, Xue X, Zappia JJ, Wise KD (1989) Batch-fabricated thinfilm electrodes for stimulation of the central auditory system. IEEE Trans Biomed Eng 36: 693-704

Bierer SM, Anderson DJ (1999) Multi-channel spike detection and sorting using an array processing technique. Neurocomputing 26-27: 947-956

Brand A, Behrend O, Marquardt T, McAlpine D, Grothe B (2002) Precise inhibition is essential for microsecond interaural time difference coding. Nature 417: 543-547

Brugge JF, Reale RA, Hind JE (1996) The structure of spatial receptive fields of neurons in primary auditory cortex of the cat. J Neurosci 16: 4420-4437

DeValois RL, DeValois KK (1993) A multi-stage color model. Vision Res 33: 1053-1065

Eggermont JJ (1998) Azimuth coding in primary auditory cortex of the cat. II. Relative latency and interspike interval representation. J Neurophysiol 80: 2151-2161

Furukawa S, Xu L, Middlebrooks JC (1998) Coding of sound source location by relative spike timing between pairs of cortical neurons. In: Abstracts of the 28th annual meeting of the Society for Neuroscience, Los Angeles, 7-12 November 1998, pp 1402

Furukawa S, Middlebrooks JC (2001) Cortical representation of auditory space: information-bearing features of spike patterns. J Neurophysiol 87: 1749-1762

Georgopoulos AP, Schwartz AB, Kettner RE (1986) Neuronal population coding of movement direction. Science 233: 14161419

Greene TC (1929) The ability to localize sound: a study of binaural hearing in patients with a tumor of the brain. Arch Surg 18: $1825-1841$

Heffner HE, Masterton RB (1975) Contribution of auditory cortex to sound localization in the monkey (Macaca mulatta). J Neurophysiol 38: 1340-1358

Heffner HE, Heffner RS (1990) Effect of bilateral auditory cortex lesions on sound localization in Japanese macaques. J Neurophysiol 64: 915-931

Hinton GE, McClelland JL, Rumelhart DE (1986) Distributed representations. In: McClelland JL, Rumelhart DE, PDP Research Group (eds) Parallel distributed processing: explorations in the microstructure of cognition. MIT Press, Cambridge, MA, pp 77-109

Huang CL, Winer JA (2000) Auditory thalamocortical projections in the cat: laminar and areal patterns of input. J Compar Neurol 427: 302-331

Jeffress LA (1948) A place theory of sound localization. J Compar Physiol Psychol 41: 35-39

Jenison RL (2001) Decoding first-spike latency: a likelihood approach. Neurocomputing 38-40: 239-248 
Jenkins WM, Masterton RB (1982) Sound localization: effects of unilateral lesions in the central auditory system. J Neurophysiol 47: $987-1016$

Jenkins WM, Merzenich MM (1984) Role of cat primary auditory cortex for sound-localization behavior. J Neurophysiol 52: 819-847

Kavanagh GL, Kelly JB (1987) Contributions of auditory cortex to sound localization by the ferret (Mustela putorius). J Neurophysiol 57: 1746-1766

Kitzes LM, Hollrigel GS (1996) Response properties of units in the posterior auditory field deprived of input from the ipsilateral primary auditory cortex. Hear Res 100: 120-130

Klingon GH, Bontecou DC (1966) Localization in auditory space. Neurology 16: 879-886

Malhotra S, Hall A, Manafov E, Woller E, Lomber S (2003) Cerebral areas mediating sound localization in the cat: cooling deactivation of 19 cortical loci. In: Abstracts of the 33rd annual meeting of the Society for Neuroscience, New Orleans, 8-12 November 2003

Marquardt T, McAlpine D (2001) Simulation of binaural unmasking using just four binaural channels. In: Assoc Res Otolaryngol Abstr 24: 87

McAlpine D, Jiang D, Palmer AR (2001) A neural code for lowfrequency sound localization in mammals. Nat Neurosci 4: 396-401

Mickey BJ, Middlebrooks JC (2003) Representation of auditory space by cortical neurons. J. Neurosci. 23: 8649-8663

Middlebrooks JC, Dykes RW, Merzenich MM (1980) Binaural response-specific bands in primary auditory cortex (AI) of the cat: topographical organization orthogonal to isofrequency contours. Brain Res 181: 31-48

Middlebrooks JC, Zook JM (1983) Intrinsic organization of the cat's medial geniculate body identified by projections to binaural response-specific bands in the primary auditory cortex. J Neurosci 3: 203-224

Middlebrooks JC, Knudsen EI (1984) A neural code for auditory space in the cat's superior colliculus. J Neurosci 4: 2621-2634
Middlebrooks JC, Xu L, Eddins AC, Green DM (1998) Codes for sound-source location in nontonotopic auditory cortex. J Neurophysiol 80: 863-881

Palmer AR, King AJ (1982) A monaural space map in the guineapig superior colliculus. Hear Res 17: 267-280

Rauschecker JP (1998) Parallel processing in the auditory cortex of primates. Audiol Neuro-otol 3: 86-103

Rauschecker JP, Tian B (2000) Mechanisms and streams for processing of "what" and "where" in auditory cortex. Proc Natl Acad Sci USA 97(22): 11800-11806

Reale RA, Imig TJ (1980) Tonotopic organization in auditory cortex of the cat. J Compar Neurol 192: 265-291

Rose JE, Woolsey CN (1949) The relations of thalamic connections, cellular structure, and evocable activity in the auditory region of the cat. J Compar Neurol 91: 441-466

Rouiller EM, Simm GM, Villa AEP, de Ribaupierre Y, de Ribaupierre F (1991) Auditory corticocortical interconnections in the cat: evidence for parallel and hierarchical arrangement of the auditory cortical areas. Exp Brain Res 86: 483-505

Sanchez-Longo LP, Forster FM (1958) Clinical significance of impairment of sound localization. Neurology 8: 119-125

Stecker GC, Harrington IA, Macpherson EA, Middlebrooks JC (2003a) Spatial sensitivity of neurons in the "dorsal zone" (area DZ) of cat auditory cortex. In: Abstracts of the 33rd annual meeting of the Society for Neuroscience, New Orleans, 8-12 November 2003

Stecker GC, Mickey BJ, Macpherson EA, Middlebrooks JC (2003b) Spatial sensitivity in field PAF of cat auditory cortex. J Neurophysiol 89: 2889-2903

Wortis SB, Pfeiffer AZ (1948) Unilateral auditory-spatial agnosia. J Nerv Ment Disord 108: 181-186

Xu L, Furukawa S, Middlebrooks JC (1998) Sensitivity to soundsource elevation in nontonotopic auditory cortex. J Neurophysiol 80: 882-894

Zatorre RJ, Penhune VB (2001) Spatial localization after excision of human auditory cortex. J Neurosci 21: 6321-6328 